\title{
$N$-碘代丁二酰亚胺促进的咪唑并[1,2-a]吡啶衍生物的芳烃硫基化反应
}

\author{
安艳妮李建晓* 李蒙李春生杨少容* \\ (华南理工大学化学与化工学院 广州 510640)
}

\begin{abstract}
摘要 以离子液体 $[\mathrm{Bmim}] \mathrm{BF}_{4}$ 为溶剂, 实现了 $N$-碘代丁二酰亚胺促进的咪唑并 $[1,2-a$ 吡啶衍生物与二芳基二硫醚发生 的氧化芳烃硫基化反应，以中等至优良产率(73\% 86\%)合成系列烃硫基取代的含氮环化合物. 其结构均经 $\mathrm{IR}$, ${ }^{1} \mathrm{H}$ $\mathrm{NMR},{ }^{13} \mathrm{C} \mathrm{NMR}$ 及 HRMS 确证. 该反应具有反应条件温和、底物适用范围广、环境友好等优点, 可为含有烃硫基取代 的天然产物及复杂药物分子的合成提供潜在的合成途径.
\end{abstract}

关键词 $N$-碘代丁二酰亚胺; 离子液体; 咪唑并 $[1,2-a]$ 吡啶; 二芳基二硫醚; 芳烃硫基化反应

\section{$N$-lodosuccinimide-Promoted Regioselective Arylsulfenylation of Imidazo[1,2-a]pyridines with Disulfides}

\author{
An, Yanni Li, Jianxiao* Li, Meng Li, Chunsheng Yang, Shaorong* \\ (School of Chemistry and Chemical Engineering, South China University of Technology, Guangzhou 510640)
}

\begin{abstract}
An efficient and practical $N$-iodosuccinimide-promoted regioselective arylsulfenylation of imidazo[1,2- $a$ ]pyridines with disulfides has been developed to afford functionalized 3-sulfenylimidazo[1,2-a]pyridines heterocycles in moderate to good yields. Their structures were confirmed by IR, ${ }^{1} \mathrm{H}$ NMR, ${ }^{13} \mathrm{C}$ NMR and HRMS. Furthermore, this method has the advantages of mild reaction conditions, broad substrates scope and environmentally benign. Notably, the current methodology could also be conveniently applied to the synthesis of thioarylated naturally occurring biologically active frameworks.
\end{abstract}

Keywords $N$-iodosuccinimide; ionic liquids; imidazo[1,2-a]pyridines; disulfides; arylsulfenylation

碳-杂原子键的形成反应是有机合成化学研究的重 要内容 ${ }^{[1]}$. 而作为一类重要的碳-杂键, 碳-硫键广泛存 在于天然产物和药物活性分子中 ${ }^{[2]}$. 因此, 发展新颖的 合成方法与策略实现碳一杂键的高效、简捷的构建在有 机合成、药物合成以及材料化学中具有重要的意义. 近 年来，诸多代表性的合成策略陆续被报道.一般来说， 主要包括以下三种: (1)烯基或者芳基卤化物与硫酚、磺 酰氯、二芳基二硫醚以及其他活化的硫代试剂的偶联反 应 ${ }^{[3]} ;$ (2)烷基或者芳基锂或者格氏试剂与硫代亚磺酸 盐、二芳基二硫醚以及单质硫的交叉偶联反应 ${ }^{[4]}$; (3)芳 香的碳(杂)环与活化的硫代试剂的亲电取代反应 ${ }^{[5]}$. 尽 管上述烃硫基化反应在构建新的、功能团化的 $\mathrm{C}-\mathrm{S}$ 键 方面取得了显著的进展, 但是在某种程度上尚存在一定 的不足, 比如硫酚具有很强的刺激性气味; 磺酰氯易水 解, 具有腐蚀性和毒性; 并且有些活化的硫代试剂稳定
性较差等.

另外, 咪唑并 $[1,2-a]$ 吡啶及其衍生物因其特殊的药 物活性及光学性能一直受到化学工作者的关注 ${ }^{[6]}$. 近年 来, 基于功能化的咪唑并 $[1,2-a]$ 吡啶衍生物的合成备受 青崃, 然而通过构建 $\mathrm{C}-\mathrm{S}$ 键构筑咪唑并 $[1,2-a$ 吡啶含 硫衍生物的报导却不多见 ${ }^{[7]} .2015$ 年, 葛泽梅课题组 ${ }^{[8]}$ 通过碘催化的咪唑并 $[1,2-a]$ 吡啶衍生物与芳基亚磺酸钠 的偶联反应高效、高区域选择性地实现芳硫基化反应; 曹华课题组 ${ }^{[9]}$ 实现了碘化亚铜催化的、氧气氛围中的氧 化烃硫基化反应; Braga 课题组 ${ }^{[10]}$ 实现作碘催化剂、二甲 基亚砜(DMSO)为氧化剂、无溶剂条件下咪唑并 [1,2- $a$ ] 吡啶衍生物与二芳基二硫梄的偶联反应. 同时, 我们课 题组 ${ }^{[11]}$ 在构建 $\mathrm{C}-\mathrm{S}$ 键方面也取得了阶段性的研究进展.

因此，我们课题组在以离子液体为反应介质的交叉 偶联反应 ${ }^{[12]}$ 以及通过串联多组分的策略构建 $\mathrm{C}-\mathrm{S}$ 键 ${ }^{[13]}$

* Corresponding author. E-mail: lisryang@scut.edu.cn

Received September 29, 2016; revised November 7, 2016; published online November 17, 2016.

Project supported by the National Natural Science Foundation of China (No. 21502055), the Fundamental Research Funds for the Central Universities (No. 2015ZM150) and the China Postdoctoral Science Foundation (Nos. 2015M572303, 2016T90779).

国家自然科学基金(No. 21502055)、中央高校基本科研业务费(No. 2015ZM150)和中国博士后科学基金(Nos. 2015M572303, 2016T90779)资助项目. 
的研究基础上, 进一步以咪唑并 $[1,2-a$ 吡啶衍生物和系 列二芳基二硫醚为底物, 在离子液体中研究了它们的交 叉偶联反应. 结果表明, 温度为 $60{ }^{\circ} \mathrm{C}$, 以 $N$-碘代丁二 酰亚胺(NIS)作催化剂, 离子液体 $[\mathrm{Bmim}] \mathrm{BF}_{4}$ 作溶剂, 以 较高产率(73\% 86\%)简捷、高效、高区域选择性地合 成系列統基化的咪唑并 $[1,2-a$ ]吡啶衍生物(Eq. 1).

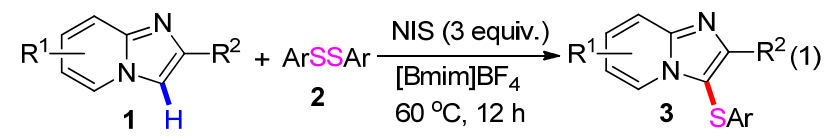

\section{1 结果与讨论}

\subsection{NIS 促进的烃硫基化反应条件优化}

为了得到优化的反应条件，反应以 2-甲基咪唑并 [1,2- $a$ 吡啶 (1a)与二苯基二硫醚(2a)为模板反应对偶联 反应进行了探究, 考察了不同的碘源、溶剂的种类以及 反应温度等对反应的影响, 具体结果如表 1 所示.

在没有碘源时, 反应是不能进行的(Entry 1). 碘源 种类的篮选表明(Entries 2 9), 当使用 NIS 作为反应的 促进剂, 反应具有较好的气相色谱(GC)转化率和较高的 区域选择性(Entry 7), 其他的碘源对反应无明显的促进 作用, 故确定 NIS 作为该条件下的最佳的反应促进剂.

紧接着, 常规溶剂的考察表明(Entries $10 \sim 15)$, DMSO 是较为合适的反应溶剂, 目标化合物 $\mathbf{3 a}$ 的 GC 收率为 $69 \%$ (Entry 10). 其他的有机溶剂, 比如 DCM、 $\mathrm{EtOH}$ 、甲苯、 $\mathrm{THF}$ 以及 $\mathrm{CH}_{3} \mathrm{CN}$, 反应的 $\mathrm{GC}$ 转化率均 出现不同程度的降低. 根据文献报道, 离子液体能够有 效地促进富电子烯烃的芳烃硫基化反应. 因此, 我们对 离子液体的种类进行了考察 ${ }^{[7 \mathrm{a}]}$. 实验发现, 咪唑型离子 液体在一定的范围内能够提高反应的 GC 转化率(Entries $16 \sim 20)$. 当使用 $\left[\mathrm{Bmim}^{-} \mathrm{BF}_{4}\right.$ 作溶剂时, 反应有较好的 $\mathrm{GC}$ 转化率和较高的区域选择性(Entry 16); 其他离子液 体, 如 $[\mathrm{Bmim}] \mathrm{PF}_{6},[\mathrm{Bmim}] \mathrm{Cl},\left[\mathrm{C}_{2} \mathrm{O}_{2} \mathrm{mim}^{-} \mathrm{BF}_{4}\right.$ 以及 $\left[\mathrm{C}_{2} \mathrm{O}-\right.$ $\mathrm{Hmim}_{\mathrm{B}} \mathrm{BF}_{4}$, 反应的 $\mathrm{GC}$ 转化率并未提高, 故认为该条件 下, $[\mathrm{Bmim}] \mathrm{BF}_{4}$ 是较为合适的离子液体. 可能的原因是 离子液体的纯离子环境有利于稳定生成的芳基硫酚正 离子, 进而高效实现芳基硫酚正离子的去质子化过程 [7a]. 实验结果表明, 该反应体系中离子液体可以循环利 用 6 次效率没有明显降低. 但是, 当使用第 7 次时活性 明显降低, 可能是因为多次反应体系副产物的积累, 导 致离子液体浓度降低, 同时反应过程副产物在一定程度 上破坏了咪唑阳离子的稳定结构, 进而致使其转化率下 降.

进一步地, 温度的考察表明(Entries 21 23), 在一 定程度上升高温度, 可以提高反应的 GC 转化率(Entry
表 1 2-甲基咪唑并 $[1,2-a]$ 吡啶(1a)与二苯基二硫醚(2a)偶联 反应的条件优化 ${ }^{a}$

Table 1 Optimization of conditions for the coupling of 2-methylimidazo[1,2- $a]$ pyridine with diphenyl disulfide

\begin{tabular}{|c|c|c|c|c|}
\hline Entry & $\begin{array}{l}\text { Iodine } \\
\text { source }\end{array}$ & Solvent & Temp. & Yield $^{b} / \%$ \\
\hline 1 & - & DMF & r.t. & - \\
\hline 2 & $\mathrm{I}_{2}$ & DMF & r.t. & 35 \\
\hline 3 & $\mathrm{ICl}$ & DMF & r.t. & 43 \\
\hline 4 & TBAI & DMF & r.t. & Trace \\
\hline 5 & KI & DMF & r.t. & 12 \\
\hline 6 & $\mathrm{NaI}$ & DMF & r.t. & Trace \\
\hline 7 & NIS & DMF & r.t. & 51 \\
\hline 8 & $\mathrm{I}_{2} \mathrm{O}_{5}$ & DMF & r.t. & Trace \\
\hline 9 & DIB & DMF & r.t. & 17 \\
\hline 10 & NIS & DMSO & r.t. & 69 \\
\hline 11 & NIS & DCM & r.t. & 14 \\
\hline 12 & NIS & $\mathrm{EtOH}$ & r.t. & 18 \\
\hline 13 & NIS & Toluene & r.t. & 46 \\
\hline 14 & NIS & THF & r.t. & 45 \\
\hline 15 & NIS & $\mathrm{CH}_{3} \mathrm{CN}$ & r.t. & 59 \\
\hline 16 & NIS & {$[\mathrm{Bmim}] \mathrm{BF}_{4}$} & r.t. & 76 \\
\hline 17 & NIS & {$[\mathrm{Bmim}] \mathrm{PF}_{6}$} & r.t. & 62 \\
\hline 18 & NIS & {$[\mathrm{Bmim}] \mathrm{Cl}$} & r.t. & 60 \\
\hline 19 & NIS & {$\left[\mathrm{C}_{2} \mathrm{O}_{2} \mathrm{mim}\right] \mathrm{BF}_{4}$} & r.t. & 52 \\
\hline 20 & NIS & {$\left[\mathrm{C}_{2} \mathrm{OHmim}\right] \mathrm{BF}_{4}$} & r.t. & 63 \\
\hline 21 & NIS & {$[\mathrm{Bmim}] \mathrm{BF}_{4}$} & 50 & 84 \\
\hline 22 & NIS & {$[\mathrm{Bmim}] \mathrm{BF}_{4}$} & 60 & $87(80)$ \\
\hline 23 & NIS & {$[\mathrm{Bmim}] \mathrm{BF}_{4}$} & 70 & 83 \\
\hline $24^{c}$ & NIS & {$[\mathrm{Bmim}] \mathrm{BF}_{4}$} & 60 & 87 \\
\hline
\end{tabular}

${ }^{a}$ Reaction condition: 1a $(0.2 \mathrm{mmol}), \mathbf{2 a}(0.30 \mathrm{mmol})$, iodine source (3 equiv.) and solvents $(1.0 \mathrm{~mL})$ under air for $12 \mathrm{~h}$. The value in parentheses is the yield of isolated product. $\mathrm{NIS}=N$-iodosuccinimide. $\mathrm{DIB}=$ iodobenzene diacetate. $[\mathrm{Bmim}] \mathrm{BF}_{4}=1$-butyl-3- methylimidazolium tetrafluoroborate. $[\mathrm{Bmim}] \mathrm{PF}_{6}=$ 1-butyl-3-methylimidaz- olium hexafluorophosphate. $[\mathrm{Bmim}] \mathrm{Cl}=1$-butyl-3methylimidazolium chloride. $\left[\mathrm{C}_{2} \mathrm{O}_{2}\right.$ mim $] \mathrm{BF}_{4}=1$-carboxymethyl-3-methylimidazolium tetrafluoroborate. $\left[\mathrm{C}_{2} \mathrm{OHmim}\right] \mathrm{BF}_{4}=1$-hydroxyethyl-3-methylimidazolium tetrafluoroborate. ${ }^{b} \mathrm{GC}$ yield. ${ }^{c} 24 \mathrm{~h}$.

22); 相反地, 继续升高温度达到 $70{ }^{\circ} \mathrm{C}$ 时, 反应的 $\mathrm{GC}$ 转化率并没有改善, 故认为温度为 $60{ }^{\circ} \mathrm{C}$ 是较为合适的 反应温度. 最后, 延长反应时间至 $24 \mathrm{~h}$, 反应的 $\mathrm{GC}$ 转化 率并没有提高(Entry 24).

因此，优化后较合适的反应条件为：以 $\left[\mathrm{Bmim}^{-} \mathrm{BF}_{4}\right.$ 为反应溶剂, 3 equiv. NIS 为反应的促进剂, 反应温度为 $60{ }^{\circ} \mathrm{C}$, 反应时间为 $12 \mathrm{~h}$.

\section{2 烃硫基化反应底物普适性考察}

在优化条件下, 我们进一步考查此催化体系的底物 普适性(表 2). 从表 2 可知: 对二芳基二硫醚的考察表 明: 无论苯环上取代基是吸电子基团还是供电子基团, 
表 2 咪唑并 $\left[1,2-a\right.$ ] 吡啶与二芳基二硫醚烃硫基化反应 ${ }^{a}$

Table 2 Sulfenylation of imidazo[1,2- $a$ ]pyridines with disulfides

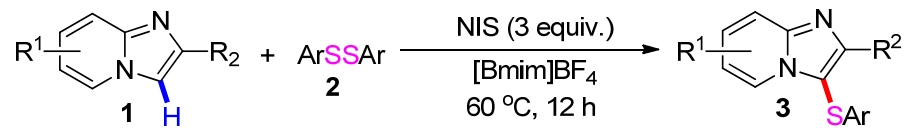

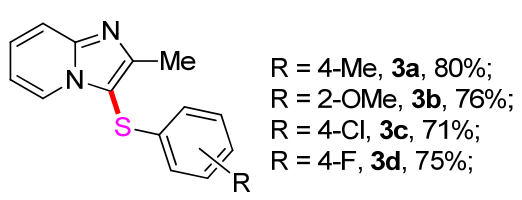

Ru-t

$\mathrm{R}=\mathrm{Me}, \mathbf{3 h}, \mathbf{8 1 \%}$

$\mathrm{R}=\mathrm{Cl}, \mathbf{3 i}, \mathbf{7 6} \%$

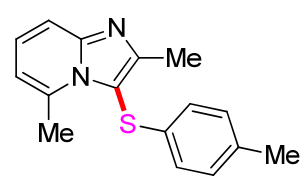

3e, $83 \%$<smiles>Cc1ccc(Sc2c(C)nc3ccc(C)cn23)cc1</smiles>

3f, $84 \%$

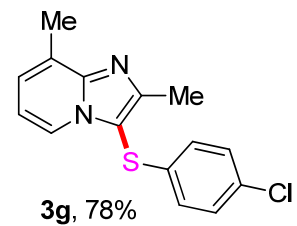

$\mathrm{R}=\mathrm{Me}, \mathbf{3} \mathbf{I}, 86 \%$ $\mathrm{R}=\mathrm{Cl}, 3 \mathrm{~m}, 79 \%$ $\mathrm{Cl} \mathrm{R}=\mathrm{Br}, \mathbf{3 n}, 78 \%$;

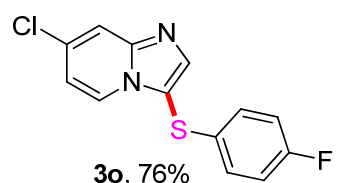

3o, $76 \%$
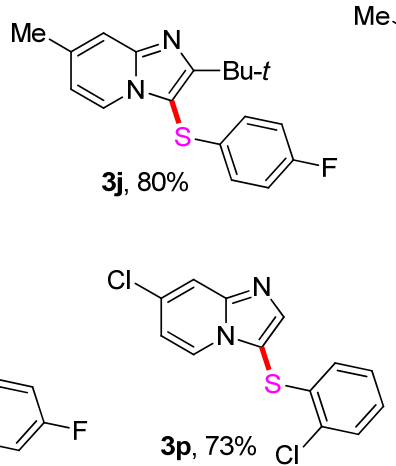
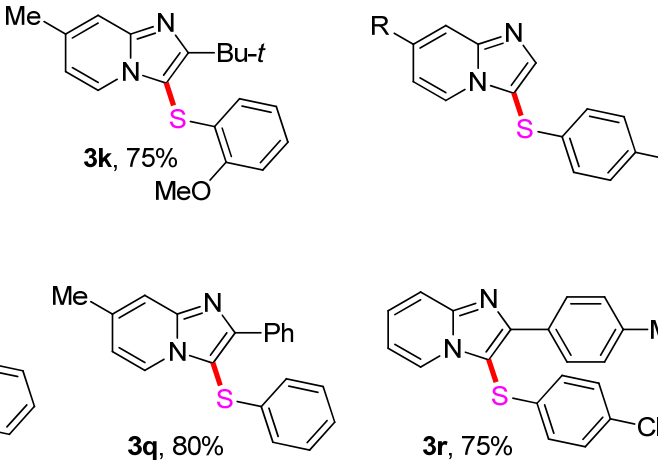

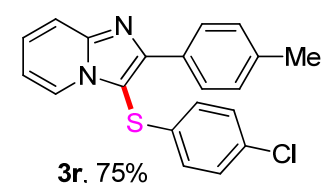

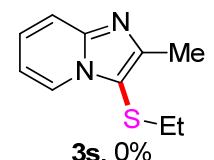

${ }^{a}$ 反应条件: 1 ( $\left.0.2 \mathrm{mmol}\right), 2(0.3 \mathrm{mmol}), \mathrm{NIS}$ (3 equiv.), [Bmim]BF $4(1.0 \mathrm{~mL})$, 反应温度为 $60{ }^{\circ} \mathrm{C}$, 反应时间为 $12 \mathrm{~h}$.

烃硫基化反应均能得到较好的转化 $(\mathbf{3 a} \sim 3 \mathbf{d})$. 并且, 苯 环连接供电子基，比如甲基、甲氧基时，反应的分离收 率略高于吸电子取代的二芳基二硫醚. 紧接着, 取代基 的位置研究发现，取代基的位置对反应的收率无显著的 影响(3e 3g); 进一步地, 咪唑并[1,2-a]吡啶环 C(2)位 取代基的考察表明，无论 $\mathrm{C}(2)$ 位是甲基、叔丁基、苯基 还是无取代基, 反应均能实现优良产率的转化(3c，3i， 3m, 3r). 另外, 当咪唑并 $[1,2-a]$ 吡啶环 C(2)和 C(3)位同 时存在较大空间位阻的芳基时，反应亦能以良好的收率 得到目标化合物 $(\mathbf{3 q}, \mathbf{3 r})$. 遗憾的是, 在优化的条件下, 烷基二硫醚并未得到目标化合物 $3 \mathrm{~s}$.

根据上述实验结果以及相关文献描述，该反应的可 能机理是: 首先, NIS 促进芳基二硫醚断裂生成亲电物 种中间体 $\mathrm{ArSI}^{[5 \mathrm{c}, 14]}, \mathrm{ArSI}$ 进攻富电子的芳香杂环化合物 生成芳基硫酚正离子, 然后去质子化可得芳香杂环硫醚 产物 ${ }^{[14]}$.

\section{4 产物结构表征}

所得化合物的结构经 IR、 ${ }^{1} \mathrm{H} N M R 、{ }^{13} \mathrm{C} N M R$ 和 HRMS 确证. 红外测试结果表明, 目标化合物波数在 $3066 \sim 3043 \mathrm{~cm}^{-1}$ 的较强吸收峰为 $\mathrm{C}=\mathrm{C}-\mathrm{H}$ 伸缩振动吸 收峰, 波数在 $1649 \sim 1620 \mathrm{~cm}^{-1}$ 的吸收峰为 $\mathrm{C}=\mathrm{C}$ 伸缩 振动吸收峰. 分析目标化合物的 ${ }^{1} \mathrm{H}$ NMR 可知, $\delta$ 为 $8.02 \sim 7.92$ 处出现的单峰为咪唑并 $[1,2-a$ ] 吡啶环 $\mathrm{C}(2)$ 位 不饱和氢的化学位移 $(\mathbf{3 I} \sim \mathbf{3 p})$.

\section{2 结论}

本文利用具有生物活性骨架的咪唑并 $[1,2-a]$ 吡啶化 合物与二芳基二硫醚为反应底物，通过 NIS 促进的烃硫 基化反应构建系列烃硫基取代的含氮环化合物. 研究结 果表明: 在以 $[\mathrm{Bmim}] \mathrm{BF}_{4}$ 为反应溶剂, 3 equiv. NIS 为反 应的促进剂, $60{ }^{\circ} \mathrm{C}$ 条件下, 反应 $12 \mathrm{~h}$, 各种咪唑并 [1,2- $a]$ 吡啶化合物均能与二芳基二硫醚衍生物以中等至 优良的产率得到目标化合物, 而且反应表现出较好的区 域选择性. 另外, 该反应条件温和, 易于操作和处理, 可为具有烃硫基取代的含氮环化合物的合成提供简便 的途径.

\section{3 实验部分}

\section{1 仪器与试剂}

美国 PERKIN-ELIMER 1730 FT-IR 红外光谱仪, $\mathrm{KBr}$ 压片; 德国 Bruker 公司 $400 \mathrm{MHz}$ DRX-400 核磁共 振仪，溶剂 $\mathrm{CDCl}_{3}$, 内标 TMS; 美国 LCQ Deca XP MAX 液相色谱-质谱联用仪(或美国 FINNIGAN TRACE DSQ 质谱仪, $70 \mathrm{eV}$ ); 河南省予华仪器有限公司 X-5 型显微 熔点仪.

所有试剂均为市售分析纯，未经进一步纯化. 离子 液 体 $\left([\mathrm{Bmim}] \mathrm{BF}_{4}{ }^{[15]},[\mathrm{Bmim}] \mathrm{PF}_{6}{ }^{[16]},\left[\mathrm{C}_{2} \mathrm{OHmim}\right] \mathrm{BF}_{4}{ }^{[17]}\right.$, $\left.\left[\mathrm{C}_{2} \mathrm{O}_{2} \operatorname{mim}\right] \mathrm{BF}_{4}{ }^{[17]}\right)$ 参考相应文献合成. 


\section{2 实验方法}

$25 \mathrm{~mL}$ 烧瓶中加入 $0.2 \mathrm{mmol}$ 咪唑并[1,2- $a$ ]吡啶(1)、 $0.3 \mathrm{mmol}$ 二芳基二硫醚(2)、3 equiv. NIS、 $1 \mathrm{~mL}$ 离子液 体 $[\mathrm{Bmim}] \mathrm{BF}_{4}$, 温度为 $60{ }^{\circ} \mathrm{C}$ 条件下, 反应 $12 \mathrm{~h}$. 反应结 束后, 往溶液中加入 $10 \mathrm{~mL}$ 乙酸乙酯 $(10 \mathrm{~mL} \times 3)$, 萃 取, 有机层无水硫酸镁干燥, 减压旋干得粗品, 柱层析 得 3. 目标化合物的结构经 IR, ${ }^{1} \mathrm{HNMR},{ }^{13} \mathrm{C} \mathrm{NMR}$ 和 HRMS 确证.

2-甲基-3-(4-甲基苯硫基)味唑并 $[1,2-a$ 吡啶(3a): 产 率 $80 \% .{ }^{1} \mathrm{H} \mathrm{NMR}\left(400 \mathrm{MHz}, \mathrm{CDCl}_{3}\right) \delta: 8.13$ (d, $J=6.8$ $\mathrm{Hz}, 1 \mathrm{H}), 7.57$ (d, $J=9.0 \mathrm{~Hz}, 1 \mathrm{H}), 7.20$ (t, $J=7.6 \mathrm{~Hz}, 1 \mathrm{H})$, $6.97(\mathrm{~d}, J=7.6 \mathrm{~Hz}, 2 \mathrm{H}), 6.85$ (d, J=7.6 Hz, 2H), 6.75 (t, $J=6.8 \mathrm{~Hz}, 1 \mathrm{H}), 2.59$ (s, 3H), $2.22(\mathrm{~s}, 3 \mathrm{H}) ;{ }^{13} \mathrm{C} \mathrm{NMR}(100$ $\left.\mathrm{MHz}, \mathrm{CDCl}_{3}\right) \delta: 146.8,135.9,131.8,130.0,126.0,125.9$, $124.3,116.9,112.5,108.0,20.8,14.0$; IR (KBr) v: 3056, 2952, 2832, 1646, 1488, 1463, $1239 \mathrm{~cm}^{-1}$; HRMS (ESI) calcd for $\mathrm{C}_{15} \mathrm{H}_{15} \mathrm{~N}_{2} \mathrm{~S}[\mathrm{M}+\mathrm{H}]^{+}: 255.0950$, found 255.0948.

3-(2-甲氧基苯硫基)-2-甲基咪唑并 $[1,2-a$ 吡啶(3b): 产率 76\%. ${ }^{1} \mathrm{H}$ NMR (400 MHz, $\mathrm{CDCl}_{3}$ ) $\delta: 8.17$ (d, $J=6.8$ $\mathrm{Hz}, 1 \mathrm{H}), 7.60$ (d, $J=8.8 \mathrm{~Hz}, 1 \mathrm{H}), 7.26(\mathrm{t}, J=8.0 \mathrm{~Hz}, 1 \mathrm{H})$, 7.10 (t, $J=7.6 \mathrm{~Hz}, 1 \mathrm{H}), 6.87$ (d, $J=8.0 \mathrm{~Hz}, 1 \mathrm{H}), 6.80(\mathrm{t}$, $J=6.8 \mathrm{~Hz}, 1 \mathrm{H}), 6.69$ (t, $J=7.6 \mathrm{~Hz}, 1 \mathrm{H}), 6.32$ (d, $J=7.8$ $\mathrm{Hz}, 1 \mathrm{H}), 3.93$ (s, 3H), 2.56 (s, 3H); ${ }^{13} \mathrm{C} \mathrm{NMR}(100 \mathrm{MHz}$, $\left.\mathrm{CDCl}_{3}\right) \delta: 156.0,151.7,147.0,126.9,125.8,125.7,124.5$, $123.6,121.3,116.8,112.4,110.8,106.3,55.8,13.9$; IR (KBr) v: 3062, 2917, 1646, 1589, 1484, $1007 \mathrm{~cm}^{-1}$; HRMS (ESI) calcd for $\mathrm{C}_{15} \mathrm{H}_{15} \mathrm{~N}_{2} \mathrm{OS}[\mathrm{M}+\mathrm{H}]^{+}: 271.0900$, found 271.0903.

3-(4-氯苯硫基)-2-甲基咪唑并 $[1,2-a]$ 吡啶(3c): 产率 $71 \%$; ${ }^{1} \mathrm{H} \mathrm{NMR}\left(400 \mathrm{MHz}, \mathrm{CDCl}_{3}\right) \delta: 8.14(\mathrm{~d}, J=6.8 \mathrm{~Hz}$, $1 \mathrm{H}), 7.62(\mathrm{~d}, J=9.0 \mathrm{~Hz}, 1 \mathrm{H}), 7.34 \sim 7.27(\mathrm{~m}, 1 \mathrm{H}), 7.17(\mathrm{~d}$, $J=8.4 \mathrm{~Hz}, 2 \mathrm{H}), 6.86(\mathrm{~d}, J=8.0 \mathrm{~Hz}, 3 \mathrm{H}), 2.58(\mathrm{~s}, 3 \mathrm{H}) ;{ }^{13} \mathrm{C}$ NMR (100 MHz, $\left.\mathrm{CDCl}_{3}\right) \delta: 151.4,145.9,134.4,131.6$, $129.2,129.1,126.6,122.5,121.8,116.3,106.1,13.8$; IR (KBr) v: 3049, 2920, 1645, 1491, 1468, $770 \mathrm{~cm}^{-1}$; HRMS (ESI) calcd for $\mathrm{C}_{14} \mathrm{H}_{12} \mathrm{ClN}_{2} \mathrm{~S}[\mathrm{M}+\mathrm{H}]^{+}: 275.0404$, found 275.0400 .

3-(4-氟苯硫基)-2-甲基咪唑并 $[1,2-a$ 吡啶(3d): 产率 $75 \%$. ${ }^{1} \mathrm{H}$ NMR (400 MHz, $\left.\mathrm{CDCl}_{3}\right) \delta: 8.16(\mathrm{~d}, J=6.0 \mathrm{~Hz}$, $1 \mathrm{H}), 7.66 \sim 7.55(\mathrm{~m}, 1 \mathrm{H}), 7.33 \sim 7.28(\mathrm{~m}, 1 \mathrm{H}), 6.90(\mathrm{ddd}$, $J=16.8,12.4,5.4 \mathrm{~Hz}, 5 \mathrm{H}), 2.59$ (s, 3H); ${ }^{13} \mathrm{C}$ NMR $(100$ $\left.\mathrm{MHz}, \mathrm{CDCl}_{3}\right) \delta: 161.5(\mathrm{~d}, J=245.9 \mathrm{~Hz}), 151.5,146.9$, 130.5, 127.8 (d, $J=7.7 \mathrm{~Hz}), 126.1,124.1,117.0,116.4$ (d, $J=22.4 \mathrm{~Hz}$ ), 112.7, 107.7, 13.9; IR (KBr) v: 3066, 2920, 1647, 1456, 1268, $769 \mathrm{~cm}^{-1}$; HRMS (ESI) calcd for
$\mathrm{C}_{14} \mathrm{H}_{12} \mathrm{FN}_{2} \mathrm{~S}[\mathrm{M}+\mathrm{H}]^{+}: 259.0700$, found 259.0696.

2,5-二甲基-3-(4-甲苯硫基)咪唑并 [1,2- $a$ ]吡啶(3e): 产率 $83 \%$. ${ }^{1} \mathrm{H} \mathrm{NMR}\left(400 \mathrm{MHz}, \mathrm{CDCl}_{3}\right) \delta: 8.00(\mathrm{~s}, 1 \mathrm{H})$, 7.62 (d, $J=8.8 \mathrm{~Hz}, 1 \mathrm{H}), 7.17$ (d, $J=8.8 \mathrm{~Hz}, 1 \mathrm{H}), 7.03$ (d, $J=7.6 \mathrm{~Hz}, 2 \mathrm{H}), 6.88$ (d, $J=7.6 \mathrm{~Hz}, 2 \mathrm{H}), 2.59$ (s, 3H), 2.31 $(\mathrm{s}, 3 \mathrm{H}), 2.28(\mathrm{~s}, 3 \mathrm{H}) ;{ }^{13} \mathrm{C} \mathrm{NMR}\left(100 \mathrm{MHz}, \mathrm{CDCl}_{3}\right) \delta$ : $153.5,148.5,138.2,136.5,135.3,130.1,126.2,124.9$, 115.3, 114.3, 108.4, 20.8, 20.3, 14.1; IR (KBr) v: 3043, 2914, 1646, 1463, 1416, 1266, $770 \mathrm{~cm}^{-1}$; HRMS (ESI) calcd for $\mathrm{C}_{16} \mathrm{H}_{17} \mathrm{~N}_{2} \mathrm{~S}[\mathrm{M}+\mathrm{H}]^{+}:$269.1107, found 269.1105.

2,6-二甲基-3-(4-甲苯硫基)咪唑并 [1,2- $a$ ]吡啶(3f): 产率 $84 \% .{ }^{1} \mathrm{H}$ NMR (400 MHz, $\left.\mathrm{CDCl}_{3}\right) \delta: 7.99(\mathrm{~d}, J=6.8$ $\mathrm{Hz}, 1 \mathrm{H}), 7.34$ (s, 1H), 6.97 (d, $J=7.6 \mathrm{~Hz}, 2 \mathrm{H}), 6.83$ (d, $J=$ $7.6 \mathrm{~Hz}, 2 \mathrm{H}), 6.58(\mathrm{~d}, J=6.8 \mathrm{~Hz}, 1 \mathrm{H}), 2.55(\mathrm{~s}, 3 \mathrm{H}), 2.36(\mathrm{~s}$, $3 \mathrm{H}), 2.22(\mathrm{~s}, 3 \mathrm{H}) ;{ }^{13} \mathrm{C} \mathrm{NMR}\left(100 \mathrm{MHz}, \mathrm{CDCl}_{3}\right) \delta: 151.1$, $147.2,137.1,135.8,132.2,129.9,125.9,123.5,115.5$, 115.0, 107.1, 21.2, 20.8, 13.9; IR (KBr) v: 3047, 2921, 1519, 1425, 1126, $771 \mathrm{~cm}^{-1}$; HRMS (ESI) calcd for $\mathrm{C}_{16} \mathrm{H}_{17} \mathrm{~N}_{2} \mathrm{~S}[\mathrm{M}+\mathrm{H}]^{+}:$269.1107, found 269.1102.

3-(4-氯苯硫基)-2,8-二甲基咪唑并 [1,2- $a$ 吡啶(3g): 产率 78\%. ${ }^{1} \mathrm{H} \mathrm{NMR}\left(400 \mathrm{MHz}, \mathrm{CDCl}_{3}\right) \delta: 8.01(\mathrm{~d}, J=6.8$ $\mathrm{Hz}, 1 \mathrm{H}), 7.03(\mathrm{~d}, J=6.8 \mathrm{~Hz}, 1 \mathrm{H}), 6.96 \sim 6.82(\mathrm{~m}, 4 \mathrm{H})$, $6.70(\mathrm{t}, J=6.8 \mathrm{~Hz}, 1 \mathrm{H}), 2.62(\mathrm{~s}, 3 \mathrm{H}), 2.61(\mathrm{~s}, 3 \mathrm{H}) ;{ }^{13} \mathrm{C}$ NMR $\left(100 \mathrm{MHz}, \mathrm{CDCl}_{3}\right) \delta: 150.7,147.1,130.7,127.7$, 126.8 125.0, 121.9, 116.4, 116.1, 112.6, 107.9, 16.7, 13.9; IR $(\mathrm{KBr}) v:$ 3057, 3028, 2957, 1629, 1436, 1295, 765 $\mathrm{cm}^{-1}$; HRMS (ESI) calcd for $\mathrm{C}_{15} \mathrm{H}_{14} \mathrm{ClN}_{2} \mathrm{~S}[\mathrm{M}+\mathrm{H}]^{+}$: 289.0561, found 289.0558 .

2-叔丁基-3-(4-甲苯硫基)咪唑并 $\left[1,2-a\right.$ 吡啶(3h) ${ }^{[18]}$ : 产率 $81 \%$. ${ }^{1} \mathrm{H}$ NMR (400 $\left.\mathrm{MHz}, \mathrm{CDCl}_{3}\right) \delta: 8.09(\mathrm{~d}, J=6.8$ $\mathrm{Hz}, 1 \mathrm{H}), 7.67$ (d, $J=8.8 \mathrm{~Hz}, 1 \mathrm{H}), 7.20 \sim 7.12(\mathrm{~m}, 1 \mathrm{H})$, $6.94(\mathrm{~d}, J=8.0 \mathrm{~Hz}, 2 \mathrm{H}), 6.76 \sim 6.65(\mathrm{~m}, 3 \mathrm{H}), 2.19(\mathrm{~s}, 3 \mathrm{H})$, $1.56(\mathrm{~s}, 9 \mathrm{H}) ;{ }^{13} \mathrm{C}$ NMR $\left(100 \mathrm{MHz}, \mathrm{CDCl}_{3}\right) \delta: 162.0,145.6$, $135.2,132.3,129.9,125.5,124.9,123.7,117.2,112.5$, 105.0), 33.9, 30.5, 20.8; IR (KBr) v: 3056, 2936, 1628, 1506, 1425, 1126, $698 \mathrm{~cm}^{-1}$; HRMS (ESI) calcd for $\mathrm{C}_{15} \mathrm{H}_{14} \mathrm{ClN}_{2} \mathrm{~S}[\mathrm{M}+\mathrm{H}]^{+}: 289.0561$, found 289.0558.

2-叔丁基-3-(4-氯苯硫基)咪唑并 $[1,2-a]$ 吡啶 $(3 \mathbf{i})^{[18]}$ : 产率 76\%. ${ }^{1} \mathrm{H} \mathrm{NMR}\left(400 \mathrm{MHz}, \mathrm{CDCl}_{3}\right) \delta: 8.10$ (d, $J=6.8$ $\mathrm{Hz}, 1 \mathrm{H}), 7.70$ (d, $J=9.0 \mathrm{~Hz}, 1 \mathrm{H}), 7.25$ (t, $J=8.0 \mathrm{~Hz}, 1 \mathrm{H})$, $7.13(\mathrm{~d}, J=8.0 \mathrm{~Hz}, 2 \mathrm{H}), 6.78$ (dd, $J=12.4,7.2 \mathrm{~Hz}, 3 \mathrm{H})$, $1.54(\mathrm{~s}, 9 \mathrm{H}) ;{ }^{13} \mathrm{C}$ NMR $\left(100 \mathrm{MHz}, \mathrm{CDCl}_{3}\right) \delta: 162.6,145.8$, $134.6,131.4,129.4,126.0,125.9,123.6,117.5,112.9$, 104.0, 34.0, 30.4; IR (KBr) v: 3044, 2930, 1649, 1490, 1467, 1059, $800 \mathrm{~cm}^{-1}$; HRMS (ESI) calcd for $\mathrm{C}_{17} \mathrm{H}_{18^{-}}$ 
$\mathrm{ClN}_{2} \mathrm{~S}[\mathrm{M}+\mathrm{H}]^{+}: 317.0874$, found 317.0872 .

2-叔丁基-3-(4-氟苯硫基)-7-甲基咪唑并 [1,2- $a$ 吡啶 $(\mathbf{3 j})^{[18]}$ : 产率 $80 \% .{ }^{1} \mathrm{H} \mathrm{NMR}\left(400 \mathrm{MHz}, \mathrm{CDCl}_{3}\right) \delta$ : $7.99(\mathrm{~d}$, $J=6.8 \mathrm{~Hz}, 1 \mathrm{H}), 7.45(\mathrm{~s}, 1 \mathrm{H}), 6.85$ (dt, $J=13.4,8.4 \mathrm{~Hz}$, $4 \mathrm{H}), 6.61(\mathrm{~d}, J=6.8 \mathrm{~Hz}, 1 \mathrm{H}), 2.37$ (s, 3H), 1.53 (s, 9H); ${ }^{13} \mathrm{C}$ NMR $\left(100 \mathrm{MHz}, \mathrm{CDCl}_{3}\right) \delta: 162.2,161.1(\mathrm{~d}, J=245.2$ $\mathrm{Hz}), 146.1,136.9,131.3$ (d, $J=3.1 \mathrm{~Hz}), 126.4$ (d, $J=7.8$ $\mathrm{Hz}), 122.8,116.4,116.1(\mathrm{~d}, J=15.5 \mathrm{~Hz}), 115.2,103.8$, 33.9, 30.4, 21.2; IR (KBr) v: 3048, 2936, 1628, 1506, 1425, 1126, $698 \mathrm{~cm}^{-1}$; HRMS (ESI) calcd for $\mathrm{C}_{18} \mathrm{H}_{20} \mathrm{FN}_{2} \mathrm{~S}$ $[\mathrm{M}+\mathrm{H}]^{+}: 315.1326$, found 315.1322 .

2-叔丁基-3-(2-甲氧基苯硫基)-7-甲基咪唑并 [1,2- $a$ ] 吡啶(3k): 产率 75\%. ${ }^{1} \mathrm{H}$ NMR (400 $\left.\mathrm{MHz}, \mathrm{CDCl}_{3}\right) \delta: 7.95$ (d, $J=6.8 \mathrm{~Hz}, 1 \mathrm{H}), 7.45$ (s, 1H), 7.02 (t, $J=7.6 \mathrm{~Hz}, 1 \mathrm{H})$, $6.83(\mathrm{~d}, J=8.0 \mathrm{~Hz}, 1 \mathrm{H}), 6.62(\mathrm{t}, J=7.6 \mathrm{~Hz}, 1 \mathrm{H}), 6.54$ (d, $J=6.8 \mathrm{~Hz}, 1 \mathrm{H}), 6.11(\mathrm{~d}, J=7.6 \mathrm{~Hz}, 1 \mathrm{H}), 3.91(\mathrm{~s}, 3 \mathrm{H}), 2.34$ $(\mathrm{s}, 3 \mathrm{H}), 1.53(\mathrm{~s}, 9 \mathrm{H}) ;{ }^{13} \mathrm{C} \mathrm{NMR}\left(100 \mathrm{MHz}, \mathrm{CDCl}_{3}\right) \delta$ : $162.4,155.5,146.2,136.6,126.1,124.6,124.3,123.1$, $121.3,115.8,114.9,110.5,102.5,55.7,33.8,30.4,21.1$; IR (KBr) v: 3046, 2929, 1620, 1466, 1414, 1138, $702 \mathrm{~cm}^{-1}$; HRMS (ESI) calcd for $\mathrm{C}_{19} \mathrm{H}_{23} \mathrm{~N}_{2} \mathrm{OS}[\mathrm{M}+\mathrm{H}]^{+}: 327.1526$, found 327.1523 .

3-(4-氯苯硫基)-7-甲基咪唑并 $[1,2-a]$ 吡啶(3I) ${ }^{[18]}$ : 产 率 86\%. ${ }^{1} \mathrm{H}$ NMR (400 MHz, $\left.\mathrm{CDCl}_{3}\right) \delta: 8.00$ (d, $J=6.8$ $\mathrm{Hz}, 1 \mathrm{H}), 7.92$ (s, 1H), 7.45 (s, 1H), 7.11 (d, $J=8.4 \mathrm{~Hz}$, $2 \mathrm{H}), 6.89$ (d, $J=8.4 \mathrm{~Hz}, 2 \mathrm{H}), 6.66(\mathrm{~d}, J=6.8 \mathrm{~Hz}, 1 \mathrm{H}), 2.38$ $(\mathrm{s}, 3 \mathrm{H}) ;{ }^{13} \mathrm{C} \mathrm{NMR}\left(100 \mathrm{MHz}, \mathrm{CDCl}_{3}\right) \delta: 142.4,137.3$, 134.1, 131.9, 129.2 (2C), 127.2 (2C), 123.2, 116.6, 115.8, 21.2; IR (KBr) v: 3048, 2932, 1648, 1500, 1467, 1420, 1124, $698 \mathrm{~cm}^{-1}$; HRMS (ESI) calcd for $\mathrm{C}_{14} \mathrm{H}_{12} \mathrm{ClN}_{2} \mathrm{~S}[\mathrm{M}+$ $\mathrm{H}]^{+}:$275.0404, found 275.0401 .

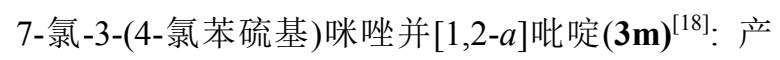
率 79\%. ${ }^{1} \mathrm{H}$ NMR (400 MHz, $\mathrm{CDCl}_{3}$ ) $\delta: 8.21$ (s, 1H), 7.99 (s, 1H), 7.64 (d, $J=9.6 \mathrm{~Hz}, 1 \mathrm{H}), 7.25$ (d, $J=9.6 \mathrm{~Hz}, 1 \mathrm{H})$, $7.15(\mathrm{~d}, J=8.0 \mathrm{~Hz}, 2 \mathrm{H}), 6.93(\mathrm{~d}, J=8.0 \mathrm{~Hz}, 2 \mathrm{H}) ;{ }^{13} \mathrm{C}$ NMR (100 MHz, $\left.\mathrm{CDCl}_{3}\right) \delta: 146.4,143.2,133.1,132.5$, $129.5,127.6,127.5,122.1,121.9,118.6,111.2$; IR (KBr) $v$ : 3049, 2918, 1645, 1489, 1406, $770 \mathrm{~cm}^{-1}$; HRMS (ESI) calcd for $\mathrm{C}_{13} \mathrm{H}_{9} \mathrm{Cl}_{2} \mathrm{~N}_{2} \mathrm{~S}[\mathrm{M}+\mathrm{H}]^{+}:$294.9858, found 294.9853.

7-溴-3-(4-氯苯硫基)咪唑并 $[1,2-a]$ 吡啶 $(\mathbf{3 n})^{[18]}$ : 产 率 78\%. ${ }^{1} \mathrm{H}$ NMR (400 MHz, $\mathrm{CDCl}_{3}$ ) $\delta: 8.32(\mathrm{~s}, 1 \mathrm{H}), 7.98$ (s, 1H), 7.59 (d, $J=9.4 \mathrm{~Hz}, 1 \mathrm{H}), 7.35$ (d, $J=9.2 \mathrm{~Hz}, 1 \mathrm{H})$, $7.17(\mathrm{~d}, J=8.2 \mathrm{~Hz}, 2 \mathrm{H}), 6.93(\mathrm{~d}, J=8.2 \mathrm{~Hz}, 2 \mathrm{H}) ;{ }^{13} \mathrm{C}$ NMR (100 MHz, $\left.\mathrm{CDCl}_{3}\right) \delta: 143.1,133.1,132.6,129.7$,
129.5 (2C), 127.6 (2C), 124.3, 118.9, 108.5; IR (KBr) v: 3046, 2933, 1626, 1502, 1459, 1425, 1132, $699 \mathrm{~cm}^{-1}$; HRMS (ESI) calcd for $\mathrm{C}_{13} \mathrm{H}_{9} \mathrm{BrClN}_{2} \mathrm{~S}[\mathrm{M}+\mathrm{H}]^{+}: 338.9353$, found 338.9350 .

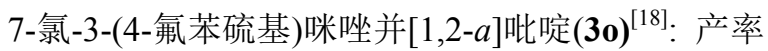
产率 76\%. ${ }^{1} \mathrm{H} \mathrm{NMR}\left(400 \mathrm{MHz}, \mathrm{CDCl}_{3}\right) \delta: 8.24(\mathrm{~s}, 1 \mathrm{H})$, 7.99 (s, 1H), 7.63 (d, $J=9.4 \mathrm{~Hz}, 1 \mathrm{H}), 7.23$ (d, $J=9.4 \mathrm{~Hz}$, $1 \mathrm{H}), 7.10 \sim 6.99(\mathrm{~m}, 2 \mathrm{H}), 6.98 \sim 6.79(\mathrm{~m}, 2 \mathrm{H}) ;{ }^{13} \mathrm{C} \mathrm{NMR}$ $\left(100 \mathrm{MHz}, \mathrm{CDCl}_{3}\right) \delta: 161.7(\mathrm{~d}, J=247.0 \mathrm{~Hz}), 146.3$, 142.9, 129.4 (d, $J=3.3 \mathrm{~Hz}), 128.7$ (d, $J=8.1 \mathrm{~Hz}), 127.4$, $122.0,121.8,118.5,116.5$ (d, $J=22.3 \mathrm{~Hz}), 112.1$; IR $(\mathrm{KBr}) v:$ 3052, 2928, 1628, 1514, 1403, 1136, $708 \mathrm{~cm}^{-1}$; HRMS (ESI) calcd for $\mathrm{C}_{13} \mathrm{H}_{9} \mathrm{ClFN}_{2} \mathrm{~S}[\mathrm{M}+\mathrm{H}]^{+}: 279.0154$, found 279.0152 .

7-氯-3-(2-氯苯硫基)味唑并 $[1,2-a]$ 吡啶 $(\mathbf{3 p})^{[18]}$ : 产 率 73\%. ${ }^{1} \mathrm{H}$ NMR (400 MHz, $\mathrm{CDCl}_{3}$ ) $\delta: 8.23$ (s, 1H), 8.02 (s, 1H), $7.68(\mathrm{~d}, J=9.2 \mathrm{~Hz}, 1 \mathrm{H}), 7.37$ (d, $J=7.8 \mathrm{~Hz}, 1 \mathrm{H})$, 7.30 (d, $J=8.8 \mathrm{~Hz}, 1 \mathrm{H}), 7.09$ (t, $J=7.6 \mathrm{~Hz}, 1 \mathrm{H}), 6.99$ (t, $J=7.6 \mathrm{~Hz}, 1 \mathrm{H}), 6.37(\mathrm{~d}, J=7.6 \mathrm{~Hz}, 1 \mathrm{H}) ;{ }^{13} \mathrm{C}$ NMR $(100$ $\left.\mathrm{MHz}, \mathrm{CDCl}_{3}\right) \delta: 143.8,134.1,131.2,130.1$ (2C), 127.8, $127.5,127.2,126.2$ (2C), 122.3, 122.1, 118.7; IR (KBr) $v$ : $3038,2915,1628,1500,1456,1408,1122,700 \mathrm{~cm}^{-1}$; HRMS (ESI) calcd for $\mathrm{C}_{13} \mathrm{H}_{9} \mathrm{Cl}_{2} \mathrm{~N}_{2} \mathrm{~S}[\mathrm{M}+\mathrm{H}]^{+}: 294.9858$, found 294.9852 .

6-甲基-2-苯基-3-(苯硫基)咪唑并 $\left[1,2-a\right.$ 吡啶(3q) ${ }^{[19]}$ : 产率 $80 \%$. ${ }^{1} \mathrm{H}$ NMR $\left(400 \mathrm{MHz}, \mathrm{CDCl}_{3}\right) \delta: 8.24 \sim 8.14(\mathrm{~m}$, $2 \mathrm{H}), 8.05(\mathrm{~s}, 1 \mathrm{H}), 7.62(\mathrm{~d}, J=9.2 \mathrm{~Hz}, 1 \mathrm{H}), 7.44 \sim 7.38(\mathrm{~m}$, $2 \mathrm{H}), 7.37-7.31(\mathrm{~m}, 1 \mathrm{H}), 7.22 \sim 7.17(\mathrm{~m}, 2 \mathrm{H}), 7.15(\mathrm{dd}$, $J=9.0,1.6 \mathrm{~Hz}, 1 \mathrm{H}), 7.11(\mathrm{dd}, J=8.4,6.4 \mathrm{~Hz}, 1 \mathrm{H}), 7.02 \sim$ $6.97(\mathrm{~m}, 2 \mathrm{H}), 2.27(\mathrm{~s}, 3 \mathrm{H}) ;{ }^{13} \mathrm{C} \mathrm{NMR}\left(100 \mathrm{MHz}, \mathrm{CDCl}_{3}\right) \delta$ : $151.3,146.2,135.6,133.6,129.8,129.5,128.5,128.4$, $128.3,125.9,125.5,123.0,122.2,117.0,105.7,18.4$; IR (KBr) v: 3041, 2926, 2834, 1636, 1455, 1417, 1336, 1025 $\mathrm{cm}^{-1}$; HRMS (ESI) calcd for $\mathrm{C}_{20} \mathrm{H}_{17} \mathrm{~N}_{2} \mathrm{~S}[\mathrm{M}+\mathrm{H}]^{+}$: 317.1107 , found 317.1103 .

3-(4-氯苯硫基)-2-(4-甲基苯基)咪唑并 $[1,2-a]$ 吡啶 (3r) ${ }^{[19]}$ : 产率 75\%. ${ }^{1} \mathrm{H}$ NMR (400 $\left.\mathrm{MHz}, \mathrm{CDCl}_{3}\right) \delta: 8.19 \sim$ $8.13(\mathrm{~m}, 2 \mathrm{H}), 8.02(\mathrm{~s}, 1 \mathrm{H}), 7.63(\mathrm{~d}, J=8.8 \mathrm{~Hz}, 1 \mathrm{H}), 7.46 \sim$ 7.39 (m, 2H), 7.36 (ddd, $J=7.2,3.6,1.2 \mathrm{~Hz}, 1 \mathrm{H}), 7.21 \sim$ $7.15(\mathrm{~m}, 3 \mathrm{H}), 6.94 \sim 6.88(\mathrm{~m}, 2 \mathrm{H}), 2.31(\mathrm{~s}, 3 \mathrm{H}) ;{ }^{13} \mathrm{C} \mathrm{NMR}$ $\left(100 \mathrm{MHz}, \mathrm{CDCl}_{3}\right) \delta: 151.5,146.3,134.1,133.3,131.9$, $130.0,129.6,128.6,128.5,128.2,126.7,123.2,122.0$, 117.1, 105.1, 18.4; IR (KBr) v: 3038, 2926, 1622, 1488, 1328, $1026 \mathrm{~cm}^{-1}$; HRMS (ESI) calcd for $\mathrm{C}_{20} \mathrm{H}_{16} \mathrm{ClN}_{2} \mathrm{~S}$ $[\mathrm{M}+\mathrm{H}]^{+}:$351.0717, found 351.0711. 
辅助材料(Supporting Information) 所有新化合物 ${ }^{1} \mathrm{H}$ $\mathrm{NMR}$ 和 ${ }^{13} \mathrm{C}$ NMR 谱图. 这些材料可以免费从本刊网站 (http://sioc-journal.cn/)上下载.

\section{References}

[1] (a) Li, B.-J.; Shi, Z.-J. Chem. Soc. Rev. 2012, 41, 5588.

(b) Ouyang, K.; Hao, W.; Zhang, W.-X.; Xi, Z. Chem. Rev. 2015, $115,12045$.

[2] (a) Kondo, T.; Mitsudo, T. Chem. Rev. 2000, 100, 3205.

(b) Liu, H.; Jiang, X. Chem.-Asian J. 2013, 8, 2546.

[3] (a) Sahoo, S. K.; Banerjee, A.; Chakraborty, S.; Patel, B. K. ACS Catal. 2012, 2, 544.

(b) Wu, Z.; Song, H.; Cui, X.; Pi, C.; Du, W.; Wu, Y. Org. Lett. 2013, 15, 1270 .

(c) Mao, J.; Jia, T.; Frensch, G.; Walsh, P. J. Org. Lett. 2014, 16, 5304.

[4] (a) Ham, J.; Yang, I.; Kang, H. J. Org. Chem. 2004, 69, 3236.

(b) Stoll, A. H.; Krasovskiy, A.; Knochel, P. Angew. Chem., Int. Ed. 2006, 45, 606

(c) Yonova, I. M.; Osborne, C. A.; Morrissette, N. S.; Jarvo, E. R. J. Org. Chem. 2014, 79, 1947.

[5] (a) Dai, C.; Xu, Z.; Huang, F.; Yu, Z.; Gao, Y.-F. J. Org. Chem. 2012, 77, 4414.

(b) Yang, F. L.; Tian, S. K. Angew. Chem., Int. Ed. 2013, 52, 4929.

(c) Xiao, F.; Xie, H.; Liu, S.; Deng, G. J. Adv. Synth. Catal. 2014, $356,364$.

[6] (a) Hand, E. S.; Paudler, W. W. J. Org. Chem. 1978, 43, 2900.

(b) Gudmundsson, K. S.; Johns, B. A. Bioorg. Med. Chem. Lett. 2007, 17, 2735.

(c) Gao, Y.; Yin, M. Z.; Wu, W. Q.; Huang, H. W.; Jiang, H. F. Adv. Synth. Catal. 2013, 355, 2263.

[7] (a) Gao, Z.; Zhu, X.; Zhang, R. RSC Adv. 2014, 4, 19891.

(b) Bagdi, A. K.; Mitra, S.; Ghosh, M.; Hajra, A. Org. Biomol. Chem. 2015, 13, 3314.

[8] Huang, X.; Wang, S.; Li, B.; Wang, X.; Ge, Z.; Li, R. RSC Adv. 2015, 5, 22654.

[9] Cao, H.; Chen, L.; Liu, J.; Cai, H.; Deng, H.; Chen, G.; Yan, C.; Chen, Y. RSC Adv. 2015, 5, 22356.

[10] Rafique, J.; Saba, S.; Rosario, A. R.; Braga, A. L. Chem.-Eur. J.
2016, 22, 11854.

[11] (a) Li, X.; Xu, Y.; Wu, W.; Jiang, C.; Qi, C.; Jiang, H. Chem. Eur. J. 2014, 20, 7911.

(b) Xu, Y.; Tang, X.; Hu, W.; Wu, W.; Jiang, H. Green Chem. 2014, 16,3720 .

(c) Gao, Y.; Gao, Y.; Tang, X.; Peng, J.; Hu, M.; Wu, W.; Jiang, H. Org. Lett. 2016, 18, 1158.

[12] (a) Li, J.; Yang, S.; Jiang, H.; Wu, W.; Zhao, J. J. Org. Chem. 2013, $78,12477$.

(b) Li, J.; Yang, S.; Huang, L.; Chen, H.; Jiang, H. RSC Adv. 2013, 3, 11529.

(c) Li, J.; Yang, S.; Wu, W.; Jiang, H. Chem. Commun. 2014, 50, 1381 .

(d) Li, J.; Li, C.; Yang, S.; Luo, W. Chin. J. Org. Chem. 2015, 35, 898 (in Chinese).

(李建晓, 李春生, 杨少容, 罗维, 有机化学, 2015, 35, 898.)

(e) Li, J.; Zhu, Z.; Yang, S.; Zhang, Z.; Wu, W.; Jiang, H. J. Org. Chem. 2015, 80, 3870.

(f) Li, J.; Zhang, Z.; Li, C.; Luo, W.; Yang, S. Chin. J. Org. Chem. 2015, 35, 2199 (in Chinese).

(李建晓, 张振明, 李春生, 罗维, 杨少容, 有机化学, 2015, 35, 2199.)

(g) An, Y.; Li, J.; Zhang, Z.; Li, C.; Yang, S. Chin. J. Org. Chem. 2016, 36, 2136 (in Chinese).

(安艳妮, 李建晓, 张振明, 李春生, 杨少容, 有机化学, 2015, 36, 2136.)

[13] (a) Li, J.; Li, C.; Yang, S.; An, Y.; Wu, W.; Jiang, H. J. Org. Chem. 2016, 81, 2875.

(b) Li, J.; Li, C.; Yang, S.; An, Y.; Wu, W.; Jiang, H. J. Org. Chem. 2016, 81,7771 .

[14] Huang, D.; Chen, J.; Dan, W.; Ding, J.; Liu, M.; Wu, H. Adv. Synth. Catal. 2012, 354, 2123.

[15] Park, S.; Kazlauskas, R. J. J. Org. Chem. 2001, 66, 8395.

[16] Branco, L. C.; Rosa, J. N.; Ramos, J. J. M.; Afonso, C. A. M. Chem. Eur. J. 2002, 8, 3671.

[17] Miao, C. X.; He, L. N.; Wang, J. Q.; Wang, J. L. Adv. Synth. Catal. 2009, 351, 2209.

[18] Zheng, Z.; Qi, D.; Shi, L. Catal. Commun. 2015, 66, 83.

[19] Hiebel, M.-A.; Berteina-Raboin, S. Green Chem. 2015, 17, 937.

(Zhao, X.) 